\title{
Some properties of non-linear fractional stochastic heat equations on bounded domains
}

\author{
Mohammud Foondun \\ University of Strathclyde
}

\author{
Ngartelbaye Guerngar \\ Auburn University
}

\author{
Erkan Nane \\ Auburn University
}

\begin{abstract}
Consider the following stochastic partial differential equation,

$$
\partial_{t} u_{t}(x)=\mathcal{L} u_{t}(x)+\xi \sigma\left(u_{t}(x)\right) \dot{F}(t, x),
$$

where $\xi$ is a positive parameter and $\sigma$ is a globally Lipschitz continuous function. The stochastic forcing term $\dot{F}(t, x)$ is white in time but possibly colored in space. The operator $\mathcal{L}$ is a non-local operator. We study the behaviour of the solution with respect to the parameter $\xi$, extending the results in [8] and [11].
\end{abstract}

Keywords: Stochastic fractional PDEs, large time behavior, colored noise.

AMS 2010 subject classification: Primary 60H15; Secondary: 35K57.

\section{Introduction and main results}

Stochastic Partial Differential Equations (SPDEs) have been used recently in many disciplines ranging from applied mathematics, statistical mechanics and theoretical physics to theoretical neuroscience, theory of complex chemical reactions (including polymer science), fluid dynamics and mathematical finance to quote only a few; see for example [9] and references therein.

In [8], the authors considered the following stochastic heat equation,

$$
\partial_{t} u_{t}(x)=\mathcal{L} u_{t}(x)+\xi \sigma\left(u_{t}(x)\right) \dot{F}(t, x),
$$

where $\mathcal{L}$ is the Dirichlet Laplacian on $B_{R}(0)$, the ball of radius $R$ centered at the origin. Under some appropriate conditions, it was shown that the long time behaviour of the solution is dependent on the noise level, that is on the values of $\xi$. More precisely, it was shown that for large values of $\xi$, the moments of the solution grow exponentially with time while for small values of $\xi$, the moments decay exponentially. In this paper, we extend the results of [8] by taking $\mathcal{L}$ to be a non-local operator, the generator of a killed stable process, namely $\mathcal{L}:=-\nu(-\Delta)^{\alpha / 2}$ for $0<\alpha \leqslant 2$ with zero exterior boundary conditions. We also provide some clarification and simplification of the proofs in [8]. Non-local operators are becoming increasingly important due to their wide applicability for modeling purposes. The class of equations we study can for instance be used to model particles moving in a discontinuous fashion while being subject to some branching mechanism; see for example Walsh [10]. 
Throughout this paper, the initial condition $u_{0}$ is always assumed to be a non-negative bounded deterministic function such that for some set $K \subset B_{R}(0)$, the quantity

$$
\int_{K} u_{0}(x) \mathrm{d} x
$$

is strictly positive. The function $\sigma$ will be subjected to the following condition.

Assumption 1.1. The function $\sigma$ is assumed to be a globally Lipschitz function satisfying

$$
l_{\sigma}|x| \leqslant|\sigma(x)| \leqslant L_{\sigma}|x| \quad \text { for all } x \in \mathbf{R},
$$

for some positive constants $l_{\sigma}$ and $L_{\sigma}$.

Following Walsh [10], we look at the mild solution of (1.1) satisfying the following integral equation,

$$
u_{t}(x)=\left(\mathcal{G}_{D} u_{0}\right)_{t}(x)+\xi \int_{B_{R}(0)} \int_{0}^{t} p_{D}(t-s, x, y) \sigma\left(u_{s}(y)\right) F(\mathrm{~d} s, \mathrm{~d} y)
$$

where

$$
\left(\mathcal{G}_{D} u_{0}\right)_{t}(x)=\int_{B_{R}(0)} u_{0}(y) p_{D}(t, x, y) \mathrm{d} y,
$$

and $p_{D}(t, x, y)$ denotes the heat kernel of the stable process. When the driving noise is white in space and time, existence-uniqueness considerations impose the conditions that $d=1$ and $1<\alpha<2$. When the noise term is not space-time white noise, it will be spatially correlated that is,

$$
\mathbb{E} \dot{F}(s, x) \dot{F}(t, y)=\delta_{0}(t-s) f(x, y),
$$

where the correlation function $f$ satisfies the inequality $f(x, y) \leqslant \tilde{f}(x-y)$, and $\tilde{f}$ is a locally integrable positive continuous function on $\mathbf{R}^{d} \backslash\{0\}$ satisfying the following Dalang type condition,

$$
\int_{\mathbf{R}^{d}} \frac{\hat{\tilde{f}}(\xi)}{1+|\xi|^{\alpha}} \mathrm{d} \xi<\infty
$$

where $\hat{\tilde{f}}$ denotes the Fourier transform of $\tilde{f}$; see [4]. We will impose the following nondegeneracy condition on $f$,

Assumption 1.2. There exists a constant $K_{R}$ such that

$$
\inf _{x, y \in B_{R}(0)} f(x, y) \geqslant K_{R}
$$

The above conditions on the correlation function are quite mild. Examples of correlation functions satisfying Assumption 1.2 include the Riesz kernel, Cauchy kernels and many more: See, for example, [6] and [7]. Our first set of results concerns equation (1.1) when the driving noise is space-time white noise which we denote by $\dot{W}$. In other words, we are looking at

$$
\left\{\begin{array}{l}
\partial_{t} u_{t}(x)=\mathcal{L} u_{t}(x)+\xi \sigma\left(u_{t}(x)\right) \dot{W}(t, x), \quad x \in B_{R}(0), \quad t>0 \\
u_{t}(x)=0, \quad x \in B_{R}(0)^{c}
\end{array}\right.
$$


Theorem 1.3. Let $u_{t}(x)$ be the unique solution of equation (1.3), then there exists $\xi_{0}>0$ such that for all $\xi<\xi_{0}$ and $x \in B_{R}(0)$,

$$
-\infty<\limsup _{t \rightarrow \infty} \frac{1}{t} \log \mathbb{E}\left|u_{t}(x)\right|^{2}<0
$$

Fix $\varepsilon>0$, then there exists $\xi_{1}>0$ such that for all $\xi>\xi_{1}$ and $x \in B_{R-\epsilon}(0)$,

$$
0<\liminf _{t \rightarrow \infty} \frac{1}{t} \log \mathbb{E}\left|u_{t}(x)\right|^{2}<\infty
$$

As in [8], we define the energy of the solution by the following quantity,

$$
\mathcal{E}_{t}(\xi)=\sqrt{\mathbb{E}\left\|u_{t}\right\|_{L^{2}\left(B_{R}(0)\right)}^{2}} \cdot
$$

The next corollary now follows easily from the above theorem.

Corollary 1.4. With $\xi_{0}$ and $\xi_{1}$ as in Theorem 1.3, we have

$$
-\infty<\limsup _{t \rightarrow \infty} \frac{1}{t} \log \mathcal{E}_{t}(\xi)<0 \quad \text { for all } \quad \xi<\xi_{0}
$$

and

$$
0<\liminf _{t \rightarrow \infty} \frac{1}{t} \log \mathcal{E}_{t}(\xi)<\infty \quad \text { for all } \quad \xi>\xi_{1} .
$$

Our next set of results concerns equation (1.1) with colored noise satisfying the conditions above. That is, we consider

$$
\left\{\begin{array}{l}
\partial_{t} u_{t}(x)=\mathcal{L} u_{t}(x)+\xi \sigma\left(u_{t}(x)\right) \dot{F}(t, x), \quad x \in B_{R}(0), \quad t>0 \\
u_{t}(x)=0, \quad x \in B_{R}(0)^{c} .
\end{array}\right.
$$

Theorem 1.5. Assume that $u_{t}$ is the unique solution to equation (1.5). Then there exists $\xi_{2}>0$ such that for all $\xi<\xi_{2}$ and $x \in B_{R}(0)$

$$
-\infty<\limsup _{t \rightarrow \infty} \frac{1}{t} \log \mathbb{E}\left|u_{t}(x)\right|^{2}<0 .
$$

Fix $\varepsilon>0$, then there exists $\xi_{3}>0$ such that for all $\xi>\xi_{3}$ and $x \in B_{R-\varepsilon}(0)$,

$$
0<\liminf _{t \rightarrow \infty} \frac{1}{t} \log \mathbb{E}\left|u_{t}(x)\right|^{2}<\infty .
$$

We then have the following easy consequence.

Corollary 1.6. Let $\xi_{2}$ and $\xi_{3}$ be as in Theorem 1.5, then

$$
-\infty<\limsup _{t \rightarrow \infty} \frac{1}{t} \log \mathcal{E}_{t}(\xi)<0 \quad \text { for all } \xi<\xi_{2}
$$

and

$$
0<\liminf _{t \rightarrow \infty} \frac{1}{t} \log \mathcal{E}_{t}(\xi)<\infty \quad \text { for all } \xi>\xi_{3}
$$


We end this introduction with a plan of the article. In section 2, we provide some estimates needed for the proofs of our main results which are presented in section 3 . Finally section 4 contains some extensions of our main results to higher moments and to some other non-local operators instead of the fractional Laplacian. Throughout this paper, the letter $\mathrm{c}$ with or without subscript(s) will denote a constant whose value is not important and can vary from place to place.

\section{Some estimates}

We begin this section with some estimates on heat kernel of the Dirichlet fractional Laplacian. For more information on these, see [1] and references therein.

$$
p_{D}(t, x, y) \leqslant c_{1}\left(t^{-\frac{d}{\alpha}} \wedge \frac{t}{|x-y|^{d+\alpha}}\right) .
$$

We will often use the above inequality in the form of $p_{D}(t, x, y) \leqslant c_{1} p(t, x-y)$, where $p_{t}(\cdot)$ is the heat kernel of the (unkilled) stable process.

- Fix $\epsilon>0$ and let $x, y \in B_{R-\epsilon}(0)$, then for all $t \leqslant \epsilon^{\alpha}$,

$$
p_{D}(t, x, y) \geqslant c_{2}\left(t^{-\frac{d}{\alpha}} \wedge \frac{t}{|x-y|^{d+\alpha}}\right) .
$$

- There exist $t_{0}>0$ and $\mu_{1}>0$ such that,

$$
c_{1} e^{-\mu_{1} t} \leqslant p_{D}(t, x, y) \leqslant c_{2} e^{-\mu_{1} t} \quad \text { for } \quad t \geqslant t_{0} .
$$

The upper bound is valid for any $x, y \in B_{R}(0)$ while the lower bound is valid for $x, y \in B_{R-\epsilon}(0)$ with $\epsilon>0$.

Our first lemma will be important for the white noise driven equation. The spatial dimension is restricted to $d=1$.

Lemma 2.1. There exists a constant $K_{\beta, \mu_{1}, \alpha}$ depending only on $\beta, \mu_{1}$ and $\alpha$ such that for all $\beta \in\left(0, \mu_{1}\right)$ and $x \in B_{R}(0)$, we have

$$
\int_{0}^{\infty} e^{\beta t} p_{D}(t, x, x) \mathrm{d} t \leqslant K_{\beta, \mu_{1}, \alpha} .
$$

Proof. We begin by writing

$$
\int_{0}^{\infty} e^{\beta t} p_{D}(t, x, x) \mathrm{d} t=\int_{0}^{t_{0}} e^{\beta t} p_{D}(t, x, x) \mathrm{d} t+\int_{t_{0}}^{\infty} e^{\beta t} p_{D}(t, x, x) \mathrm{d} t
$$

where $t_{0}$ is as in (2.3). Now using (2.1), we have

$$
\int_{0}^{t_{0}} e^{\beta t} p_{D}(t, x, x) \mathrm{d} t \leqslant c_{3} \int_{0}^{t_{0}} e^{\beta t} t^{-\frac{1}{\alpha}} \mathrm{d} t
$$


where we have used the fact that $d=1$. It is now clear that the above integral has an upper bound depending on $\beta$. Since $\beta<\mu_{1}$, we can use (2.3) to write

$$
\begin{aligned}
\int_{t_{0}}^{\infty} e^{\beta t} p_{D}(t, x, x) \mathrm{d} t \\
\quad \leqslant c_{5} \int_{t o}^{\infty} e^{-\left(\mu_{1}-\beta\right) t} \mathrm{~d} t \\
\leqslant \frac{c_{6}}{\mu_{1}-\beta} .
\end{aligned}
$$

Combining the estimates, we obtain the result.

Lemma 2.2. Let $\beta \in\left(0, \mu_{1}\right)$ and $x \in B_{R}(0)$. Then there exists a constant $c_{R, \alpha}$ depending on $R$ and $\alpha$ such that for all $t>0$

$$
\int_{B_{R}(0)} e^{\beta t} p_{D}(t, x, y) \mathrm{d} y \leqslant c_{R, \alpha}
$$

Proof. Fix $t_{0}$ as in (2.3). For $0<t<t_{0}$, we have

$$
\begin{aligned}
\int_{B_{R}(0)} e^{\beta t} p_{D}(t, x, y) \mathrm{d} y \\
\leqslant e^{\beta t} \int_{\mathbb{R}^{d}} p(t, x, y) \mathrm{d} y \\
\leqslant e^{\beta t_{0}}
\end{aligned}
$$

and for $t>t_{0}$ we use (2.3) to get

$$
\begin{array}{r}
\int_{B_{R}(0)} e^{\beta t} p_{D}(t, x, y) \mathrm{d} y \\
\leqslant c_{2} e^{-\left(\mu_{1}-\beta\right) t_{0}}
\end{array}
$$

The result now easily follows from the two inequalities above.

Lemma 2.3. Let $\beta \in\left(0,2 \mu_{1}\right)$. Then there exists a constant $c_{\beta, \mu_{1}}$ depending on $\beta$ and $\mu_{1}$ such that

$$
\int_{0}^{\infty} e^{\beta t} \int_{B_{R}(0) \times B_{R}(0)} p_{D}\left(t, x_{1}, y_{1}\right) p_{D}\left(t, x_{2}, y_{2}\right) f\left(y_{1}, y_{1}\right) \mathrm{d} t \mathrm{~d} y_{1} \mathrm{~d} y_{2} \leqslant c_{\beta, \mu_{1}},
$$

for all $x_{1}, x_{2}, y_{1}, y_{2} \in B_{R}(0)$.

Proof. We again use (2.3) so we fix $t_{0}$ accordingly. We begin by splitting the integral as follows,

$$
\begin{aligned}
\int_{0}^{\infty} e^{\beta t} & \int_{B_{R}(0) \times B_{R}(0)} p_{D}\left(t, x_{1}, y_{1}\right) p_{D}\left(t, x_{2}, y_{2}\right) f\left(y_{1}, y_{1}\right) \mathrm{d} t \mathrm{~d} y_{1} \mathrm{~d} y_{2} \\
& =\int_{0}^{t_{0}} e^{\beta t} \int_{B_{R}(0) \times B_{R}(0)} p_{D}\left(t, x_{1}, y_{1}\right) p_{D}\left(t, x_{2}, y_{2}\right) f\left(y_{1}, y_{1}\right) \mathrm{d} t \mathrm{~d} y_{1} \mathrm{~d} y_{2} \\
& +\int_{t_{0}}^{\infty} e^{\beta t} \int_{B_{R}(0) \times B_{R}(0)} p_{D}\left(t, x_{1}, y_{1}\right) p_{D}\left(t, x_{2}, y_{2}\right) f\left(y_{1}, y_{1}\right) \mathrm{d} t \mathrm{~d} y_{1} \mathrm{~d} y_{2} \\
& :=I_{1}+I_{2}
\end{aligned}
$$


$I_{1}$ can be bounded as follows: we use (2.1) to obtain

$$
\begin{aligned}
I_{1} & \leqslant e^{\beta t_{0}} \int_{0}^{t_{0}} e^{-\beta t} e^{\beta t} \int_{B_{R}(0) \times B_{R}(0)} p_{D}\left(t, x_{1}, y_{1}\right) p_{D}\left(t, x_{2}, y_{2}\right) f\left(y_{1}, y_{1}\right) \mathrm{d} t \mathrm{~d} y_{1} \mathrm{~d} y_{2} \\
& \leqslant e^{2 \beta t_{0}} \int_{0}^{\infty} e^{-\beta t} \int_{\mathbb{R}^{d} \times \mathbb{R}^{d}} p\left(t, x_{1}, y_{1}\right) p\left(t, x_{2}, y_{2}\right) \tilde{f}\left(y_{1}-y_{1}\right) \mathrm{d} t \mathrm{~d} y_{1} \mathrm{~d} y_{2} \\
& \leqslant c_{1} e^{2 \beta t_{0}} .
\end{aligned}
$$

The last inequality needs some justifications which are quite straightforward under the current conditions; see [6] for details. For $I_{2}$, we use (2.3) to write

$$
\begin{aligned}
I_{2} \leqslant & \int_{t_{0}}^{\infty} e^{\beta t} \sup _{y_{1}, y_{2} \in B_{R}(0)} p_{D}\left(t, x_{1}, y_{1}\right) p_{D}\left(t, x_{2}, y_{2}\right) \mathrm{d} t \int_{B_{R}(0) \times B_{R}(0)} f\left(y_{1}-y_{1}\right) \mathrm{d} y_{1} \mathrm{~d} y_{2} \\
& \leqslant c_{2} \int_{0}^{\infty} e^{-\left(2 \mu_{1}-\beta\right) t} \mathrm{~d} t .
\end{aligned}
$$

Combining the above estimates yields the result.

Lemma 2.4. Fix $\varepsilon>0$. Then, there exist $t_{0}>0$ and a constant $c_{\beta, \mu_{1}, t_{0}}$ such that for all $\beta>0$,

$$
\int_{0}^{\infty} e^{-\beta t} p_{D}\left(t, x_{1}, y_{1}\right) p_{D}\left(t, x_{2}, y_{2}\right) \mathrm{d} t \geqslant c_{\beta, \mu_{1}, t_{0}}
$$

whenever $x_{1}, x_{2}, y_{1}, y_{2} \in B_{R-\varepsilon}(0)$. The constant $c_{\beta, \mu_{1}, t_{0}}$ depends on $\beta, \mu_{1}$ and $t_{0}$.

Proof. Using (2.3), we have

$$
\begin{aligned}
\int_{0}^{\infty} e^{-\beta t} p_{D}\left(t, x_{1}, y_{1}\right) p_{D}\left(t, x_{2}, y_{2}\right) \mathrm{d} t \\
\quad \geqslant \int_{t_{0}}^{\infty} e^{-\beta t} p_{D}\left(t, x_{1}, y_{1}\right) p_{D}\left(t, x_{2}, y_{2}\right) \mathrm{d} t \\
\geqslant c_{1} \int_{t_{0}}^{\infty} e^{-\beta t} e^{-2 \mu_{1} t} \mathrm{~d} t \\
=\frac{c_{2} e^{-\left(\beta+2 \mu_{1}\right) t_{0}}}{\beta+2 \mu_{1}} .
\end{aligned}
$$

\section{Proofs of main results.}

\subsection{Proof of Theorem 1.3}

Proof of Theorem 1.3. Using (1.2) and the Walsh isometry, we have

$$
\mathbb{E}\left|u_{t}(x)\right|^{2}=\left|\left(\mathcal{G}_{D} u_{0}\right)_{t}(x)\right|^{2}+\xi^{2} \int_{0}^{t} \int_{B_{R}(0)} p_{D}^{2}(t-s, x, y) \mathbb{E}\left|\sigma\left(u_{s}(y)\right)\right|^{2} \mathrm{~d} y \mathrm{~d} s .
$$


from which we obtain

$$
\mathbb{E}\left|u_{t}(x)\right|^{2} \geqslant\left|\left(\mathcal{G}_{D} u_{0}\right)_{t}(x)\right|^{2} .
$$

Using the assumption on $u_{0}$, we have for $\epsilon>0$ small enough,

$$
\begin{aligned}
\left(\mathcal{G}_{D} u_{0}\right)_{t}(x) & =\int_{B_{R}(0)} u_{0}(y) p_{D}(t, x, y) \mathrm{d} y \\
& \geqslant \int_{B_{R-\epsilon}(0)} u_{0}(y) p_{D}(t, x, y) \mathrm{d} y \\
& \geqslant c_{1} e^{-\mu_{1} t},
\end{aligned}
$$

whenever $t \geqslant t_{0}$ with $t_{0}$ as in (2.3) and $x \in B_{R-\epsilon}(0)$. This immediately gives

$$
\liminf _{t \rightarrow \infty} \frac{1}{t} \log \mathbb{E}\left|u_{t}(x)\right|^{2}>-\infty \quad \text { for } \quad x \in B_{R-\epsilon}(0) .
$$

We now look at the upper bound. We will assume that $\beta \in\left(0,2 \mu_{1}\right)$. From (3.1) and the assumption on $\sigma$, we have

$$
\begin{aligned}
\mathbb{E}\left|u_{t}(x)\right|^{2} & \leqslant\left|\left(\mathcal{G}_{D} u_{0}\right)_{t}(x)\right|^{2}+\xi^{2} L_{\sigma}^{2} \int_{0}^{t} \int_{B_{R}(0)} p_{D}{ }^{2}(t-s, x, y) \mathbb{E}\left|u_{s}(y)\right|^{2} \mathrm{~d} y \mathrm{~d} s \\
& :=I_{1}+I_{2} .
\end{aligned}
$$

Using Lemma 2.2, we have

$$
\begin{aligned}
I_{1} & \leqslant c_{2} e^{-\beta t}\left|\int_{B_{R}(0)} e^{\frac{\beta t}{2}} p_{D}(t, x, y) \mathrm{d} y\right|^{2} \\
& \leqslant c_{2} e^{-\beta t} .
\end{aligned}
$$

We then look at the second term $I_{2}$. Using the semigroup property and Lemma 2.1, we have

$$
\begin{aligned}
I_{2} & =\xi^{2} L_{\sigma}^{2} e^{-\beta t} \int_{0}^{t} \int_{B_{R}(0)} e^{\beta(t-s)} p_{D}^{2}(t-s, x, y) e^{\beta s} \mathbb{E}\left|u_{s}(y)\right|^{2} \mathrm{~d} y \mathrm{~d} s \\
& \leqslant \xi^{2} L_{\sigma}^{2} e^{-\beta t} \sup _{t>0, x \in B_{R}(0)} e^{\beta t} \mathbb{E}\left|u_{t}(x)\right|^{2} \int_{0}^{t} \int_{B_{R}(0)} e^{\beta(t-s)} p_{D}^{2}(t-s, x, y) \mathrm{d} y \mathrm{~d} s \\
& \leqslant \xi^{2} L_{\sigma}^{2} e^{-\beta t} \sup _{t>0, x \in B_{R}(0)} e^{\beta t} \mathbb{E}\left|u_{t}(x)\right|^{2} \int_{0}^{t} e^{\beta s} p_{D}(2 s, x, x) \mathrm{d} s \\
& \leqslant K_{\beta, \mu_{1}, \alpha} \xi^{2} L_{\sigma}^{2} e^{-\beta t} \sup _{t>0, x \in B_{R}(0)} e^{\beta t} \mathbb{E}\left|u_{t}(x)\right|^{2} .
\end{aligned}
$$

Combining the above inequalities, we have

$$
\sup _{t>0, x \in B_{R}(0)} e^{\beta t} \mathbb{E}\left|u_{t}(x)\right|^{2} \leqslant c_{2}+K_{\beta, \mu_{1}, \alpha} \xi^{2} L_{\sigma}^{2} \sup _{t>0, x \in B_{R}(0)} e^{\beta t} \mathbb{E}\left|u_{t}(x)\right|^{2} .
$$

We now choose $\xi_{0}$ such that for $\xi \leqslant \xi_{0}$, we have $K_{\beta, \mu_{1}, \alpha} \xi^{2} L_{\sigma}^{2}<\frac{1}{2}$. This immediately gives

$$
\limsup _{t \rightarrow \infty} \frac{1}{t} \log \mathbb{E}\left|u_{t}(x)\right|^{2}<0 \text {. }
$$


We have thus proved the first half of the theorem. For the second half, we look at the following 'Laplace transform',

$$
I_{\beta}:=\int_{0}^{\infty} e^{-\beta t} \inf _{x \in B_{R-\epsilon}(0)} \mathbb{E}\left|u_{t}(x)\right|^{2} \mathrm{~d} x .
$$

Using the mild formulation and the condition on $\sigma$, we have

$$
\mathbb{E}\left|u_{t}(x)\right|^{2} \geqslant\left|\left(\mathcal{G}_{D} u_{0}\right)_{t}(x)\right|^{2}+\xi^{2} l_{\sigma}^{2} \int_{0}^{t} \int_{B_{R}(0)} p_{D}^{2}(t-s, x, y) \mathbb{E}\left|u_{s}(y)\right|^{2} \mathrm{~d} y \mathrm{~d} s .
$$

From the above, we have $I_{\beta} \geqslant I_{1}+I_{2}$, where $I_{1}$ and $I_{2}$ are Laplace transforms of the first and second term of the above display respectively. We look at $I_{1}$ first. Note that for fixed $\epsilon>0$,

$$
\begin{aligned}
\inf _{x \in B_{R-\epsilon}(0)}\left(\mathcal{G}_{D} u_{0}\right)_{t}(x) & \geqslant \int_{B_{R-\epsilon}(0)} u_{0}(y) p_{D}(t, x, y) \mathrm{d} y \\
& \geqslant c_{4} \inf _{x, y \in B_{R-\epsilon}(0)} p_{D}(t, x, y) .
\end{aligned}
$$

Using (2.3), for $t \geqslant t_{0}$, we have

$$
\begin{aligned}
I_{1} & \geqslant \int_{t_{0}}^{\infty} e^{-\beta t} \inf _{x \in B_{R-\epsilon}(0)}\left|\left(\mathcal{G}_{D} u_{0}\right)_{t}(x)\right|^{2} \mathrm{~d} t \\
& \geqslant \frac{c_{3} e^{-\left(\beta+2 \mu_{1}\right) t_{0}}}{\beta+2 \mu_{1}} .
\end{aligned}
$$

For the second term, we obtain

$$
\begin{aligned}
I_{2} & \geqslant \xi^{2} l_{\sigma}^{2} I_{\beta} \int_{t_{0}}^{\infty} e^{-\beta s} \inf _{x \in B_{R-\epsilon}(0)} p_{D}^{2}(s, x, y) \mathrm{d} y \\
& \geqslant c_{5} \xi^{2} l_{\sigma}^{2} I_{\beta} \frac{e^{-\left(\beta+2 \mu_{1}\right) t_{0}}}{\beta+2 \mu_{2}} .
\end{aligned}
$$

Combining the above inequalities yields

$$
I_{\beta} \geqslant \frac{c_{3} e^{-\left(\beta+2 \mu_{1}\right) t_{0}}}{\beta+2 \mu_{1}}+c_{5} \xi^{2} l_{\sigma}^{2} I_{\beta} \frac{e^{-\left(\beta+2 \mu_{1}\right) t_{0}}}{\beta+2 \mu_{2}} .
$$

We can now choose $\xi_{1}$ large enough so that for $\xi \geqslant \xi_{1}$, we have

$$
I_{\beta} \geqslant \frac{c_{3} e^{-\left(\beta+2 \mu_{1}\right) t_{0}}}{\beta+2 \mu_{1}}+2 I_{\beta}
$$

which gives us $I_{\beta}=\infty$. This proves

$$
\liminf _{t \rightarrow \infty} \frac{1}{t} \log \mathbb{E}\left|u_{t}(x)\right|^{2}>0
$$

The fact that

$$
\liminf _{t \rightarrow \infty} \frac{1}{t} \log \mathbb{E}\left|u_{t}(x)\right|^{2}<\infty
$$

easily follows from the ideas in [5]. We leave it to the reader to fill in the details. 


\subsection{Proof of Corollary 1.4}

Proof of Corollary 1.4. The proof follows essentially from Theorem 1.3 and the definition of the energy of the solution together with the following estimate

$$
\left|B_{R-\epsilon}(0)\right| \inf _{x \in B_{R-\epsilon}(0)} \mathbb{E}\left|u_{t}(x)\right|^{2} \leqslant \int_{B_{R}(0)} \mathbb{E}\left|u_{t}(x)\right|^{2} d x \leqslant\left|B_{R}(0)\right| \sup _{x \in B_{R}(0)} \mathbb{E}\left|u_{t}(x)\right|^{2} .
$$

\subsection{Proof of Theorem 1.5}

While one can expect the proof of Theorem 1.5 to follow a similar pattern to that of Theorem 1.3 , the noise term is now colored thus the proof is harder and requires a new idea. We provide the details of the proof of Theorem 1.5. The proof of Corollary 1.6 is omitted since it is similar to that of Corollary 1.4.

Proof of Theorem 1.5. Using the mild formulation of the solution and the assumption on $\sigma$, we obtain

$$
\begin{aligned}
\mathbb{E}\left|u_{t}(x)\right|^{2} & =\left|\left(\mathcal{G}_{D} u_{0}\right)_{t}(x)\right|^{2}+\xi^{2} \int_{0}^{t} \int_{B_{R}(0) \times B_{R}(0)} p_{D}\left(t-s, x, y_{1}\right) p_{D}\left(t-s, x, y_{2}\right) f\left(y_{1}, y_{2}\right) \\
& \times \mathbb{E}\left|\sigma\left(u_{s}\left(y_{1}\right)\right) \sigma\left(u_{s}\left(y_{2}\right)\right)\right| \mathrm{d} y_{1} \mathrm{~d} y_{2} \mathrm{~d} s \\
& \leqslant\left|\left(\mathcal{G}_{D} u_{0}\right) t(x)\right|^{2}+\xi^{2} L_{\sigma}^{2} \int_{0}^{t} \int_{B_{R}(0) \times B_{R}(0)} p_{D}\left(t-s, x, y_{1}\right) p_{D}\left(t-s, x, y_{2}\right) f\left(y_{1}, y_{2}\right) \\
& \times \mathbb{E}\left|u_{s}\left(y_{1}\right) u_{s}\left(y_{2}\right)\right| \mathrm{d} y_{1} \mathrm{~d} y_{2} \mathrm{~d} s \\
& =I_{1}+I_{2} .
\end{aligned}
$$

Set $\beta \in\left(0,2 \mu_{1}\right)$. Take $t_{0}$ as in $(2.3)$. As is the proof of Theorem 1.3, we have

$$
I_{1} \leqslant c_{1} e^{-\beta t} \quad \text { whenever } \quad t>t_{0} .
$$

We now bound $I_{2}$ by using Lemma 2.3 .

$$
\begin{aligned}
I_{2} & \leqslant \xi^{2} L_{\sigma}^{2} e^{-\beta t} \sup _{t>0, x \in B_{R}(0)} e^{\beta t} \mathbb{E}\left|u_{t}(x)\right|^{2} \\
& \times \int_{0}^{\infty} e^{\beta t} \int_{B_{R}(0) \times B_{R}(0)} p_{D}\left(t, x, y_{1}\right) p_{D}\left(t, x, y_{2}\right) f\left(y_{1}, y_{2}\right) \mathrm{d} y_{1} \mathrm{~d} y_{2} \mathrm{~d} t \\
& \leqslant c_{2} \xi^{2} L_{\sigma}^{2} e^{-\beta t} \sup _{t>0, x \in B_{R}(0)} e^{\beta t} \mathbb{E}\left|u_{t}(x)\right|^{2} .
\end{aligned}
$$

Using the two bounds above, we can use the arguments of the first part of the proof of Theorem 1.3 to show that

$$
\limsup _{t \rightarrow \infty} \frac{1}{t} \log \mathbb{E}\left|u_{t}(x)\right|^{2}<0 .
$$

The first part of our first theorem also gives us

$$
\liminf _{t \rightarrow \infty} \frac{1}{t} \log \mathbb{E}\left|u_{t}(x)\right|^{2}>-\infty \quad \text { for } \quad x \in B_{R-\epsilon}(0) .
$$


We now turn our attention to the final part of the proof. Fix $\beta, \epsilon>0$ and consider the following 'Laplace transform',

$$
J_{\beta}:=\int_{0}^{\infty} e^{-\beta t} \inf _{x, y \in B_{R-\varepsilon}(0)} \mathbb{E}\left|u_{t}(x) u_{t}(y)\right| \mathrm{d} t .
$$

From the mild solution, we have

$$
\begin{array}{r}
\mathbb{E}\left(u_{t}\left(x_{1}\right) u_{t}\left(x_{2}\right)\right)=\left(\mathcal{G}_{D} u_{0}\right)_{t}\left(x_{1}\right)\left(\mathcal{G}_{D} u_{0}\right)\left(x_{2}\right)+\xi^{2} \int_{0}^{t} \int_{B_{R}(0) \times B_{R}(0)} p_{D}\left(t-s, x_{1}, y_{1}\right) \\
\times p_{D}\left(t-s, x_{2}, y_{2}\right) f\left(y_{1}, y_{2}\right) \mathbb{E}\left(\sigma\left(u_{s}\left(y_{1}\right)\right) \sigma\left(u_{s}\left(y_{2}\right)\right)\right) \mathrm{d} y_{1} \mathrm{~d} y_{2} \mathrm{~d} s .
\end{array}
$$

Using the condition on $\sigma$, we have

$$
\begin{aligned}
& \mathbb{E}\left(\left|u_{t}\left(x_{1}\right) u_{t}\left(x_{2}\right)\right|\right) \geqslant\left|\left(\mathcal{G}_{D} u_{0}\right)_{t}\left(x_{1}\right)\left(\mathcal{G}_{D} u_{0}\right)\left(x_{2}\right)\right| \\
& \quad+\xi^{2} l_{\sigma}^{2} \int_{0}^{t} \int_{B_{R}(0) \times B_{R}(0)} p_{D}\left(t-s, x_{1}, y_{1}\right) p_{D}\left(t-s, x_{2}, y_{2}\right) f\left(y_{1}, y_{2}\right) \mathbb{E}\left|u_{s}\left(y_{1}\right) u_{s}\left(y_{2}\right)\right| \mathrm{d} y_{1} \mathrm{~d} y_{2} \mathrm{~d} s \\
& \quad:=J_{1}+J_{2} .
\end{aligned}
$$

We bound $J_{2}$ first by using the condition on the correlation function.

$$
\begin{aligned}
J_{2} & \geqslant \xi^{2} l_{\sigma}^{2} K_{R} \int_{0}^{t} \int_{B_{R-\epsilon}(0) \times B_{R-\epsilon}(0)} p_{D}\left(t-s, x_{1}, y_{1}\right) p_{D}\left(t-s, x_{2}, y_{2}\right) \mathbb{E}\left(\left|u_{s}\left(y_{1}\right) u_{s}\left(y_{2}\right)\right|\right) \mathrm{d} y_{1} \mathrm{~d} y_{2} \mathrm{~d} s \\
& \geqslant c_{3} \xi^{2} l_{\sigma}^{2} K_{R} \int_{0}^{t} \inf _{y_{1}, y_{2} \in B_{R-\epsilon}(0)} p_{D}\left(t-s, x_{1}, y_{1}\right) p_{D}\left(t-s, x_{2}, y_{2}\right) \mathbb{E}\left(\left|u_{s}\left(y_{1}\right) u_{s}\left(y_{2}\right)\right|\right) \mathrm{d} s .
\end{aligned}
$$

Using these estimates, we have

$$
J_{\beta} \geqslant \tilde{J}_{1}+\tilde{J}_{2}
$$

where $\tilde{J}_{1}$ and $\tilde{J}_{2}$ are the Laplace transforms of $J_{1}$ and $J_{2}$ respectively. As in the proof of Theorem 1.3, we have

$$
\begin{aligned}
\tilde{J}_{1} & \geqslant \int_{0}^{\infty} e^{-\beta t}\left|\left(\mathcal{G}_{D} u_{0}\right)_{t}\left(x_{1}\right)\left(\mathcal{G}_{D} u_{0}\right)\left(x_{2}\right)\right| \mathrm{d} t \\
& \geqslant \frac{c_{4} e^{-\left(\beta+2 \mu_{1}\right) t_{0}}}{\beta+2 \mu_{1}} \text { for } \quad x_{1}, x_{2} \in B_{R-\epsilon}(0) .
\end{aligned}
$$

$\tilde{J}_{2}$ can be estimated using Lemma 2.4 as follows.

$$
\tilde{J}_{2} \geqslant c_{4} \xi^{2} l_{\sigma}^{2} K_{R} J_{\beta} \frac{e^{-\left(\beta+2 \mu_{1}\right) t_{0}}}{\beta+2 \mu_{1}} .
$$

We therefore have

$$
J_{\beta} \geqslant \frac{c_{4} e^{-\left(\beta+2 \mu_{1}\right) t_{0}}}{\beta+\mu_{1}}+c_{4} \xi^{2} l_{\sigma}^{2} K_{R} J_{\beta} \frac{e^{-\left(\beta+2 \mu_{1}\right) t_{0}}}{\beta+2 \mu_{1}}
$$


Therefore there exists a $\xi_{3}>0$ such that we have $J_{\beta}=\infty$ for $\xi \geqslant \xi_{3}$. Using the ideas above, we have

$$
\int_{0}^{\infty} e^{-\beta t} \mathbb{E}\left|u_{t}(x)\right|^{2} \mathrm{~d} t \geqslant c_{5} K_{R} J_{\beta} \frac{e^{-\left(\beta+2 \mu_{1}\right) t_{0}}}{\beta+2 \mu_{1}} \quad \text { for } \quad x \in B_{R-\epsilon}(0) .
$$

Therefore for $\xi \geqslant \xi_{3}$, we obtain

$$
\int_{0}^{\infty} e^{-\beta t} \mathbb{E}\left|u_{t}(x)\right|^{2} \mathrm{~d} t=\infty
$$

which implies that

$$
\liminf _{t \rightarrow \infty} \frac{1}{t} \log \mathbb{E}\left|u_{t}(x)\right|^{2}>0 \quad \text { for } \quad x \in B_{R-\epsilon}(0) . .
$$

Again the ideas of [6] give

$$
\liminf _{t \rightarrow \infty} \frac{1}{t} \log \mathbb{E}\left|u_{t}(x)\right|^{2}<\infty .
$$

The theorem is therefore proved.

\section{Some extensions}

We conclude this paper with some extensions that can be proved using the methods developed in our paper. Since our main theorems (Theorem $1.3 \&$ Theorem 1.5 ) are about second moments of the solution to the corresponding equation, one may naturally ask if they also hold for higher moments. This is actually answered in the following theorems.

Theorem 4.1. If $u_{t}$ is the unique solution to (1.3), then for all $p \geqslant 2$, there exists $\xi_{0}(p)>0$ such that for all $\xi<\xi_{0}(p)$ and $x \in B_{R}(0)$,

$$
-\infty<\limsup _{t \rightarrow \infty} \frac{1}{t} \log \mathbb{E}\left|u_{t}(x)\right|^{p}<0
$$

On the other hand, for all $\varepsilon>0$, there exists $\xi_{1}(p)>0$ such that for all $\xi>\xi_{1}(p)$ and $x \in B_{R-\epsilon}(0)$,

$$
0<\liminf _{t \rightarrow \infty} \frac{1}{t} \log \mathbb{E}\left|u_{t}(x)\right|^{p}<\infty .
$$

The proof of this theorem follows from the Burkhölder-Davis-Gundy inequality for the upper bound and Jensen's inequality for the lower bound. We do not provide a proof here. The reader can refer to [8] for details. Next we state a result similar to Theorem 4.1 in higher dimension.

Theorem 4.2. Let $u_{t}(x)$ be the unique solution to (1.5), then for all $p \geqslant 2$ there exists $\xi_{2}(p)>0$ such that for all $\xi<\xi_{2}(p)$ and $x \in B_{R}(0)$

$$
-\infty<\limsup _{t \rightarrow \infty} \frac{1}{t} \log \mathbb{E}\left|u_{t}(x)\right|^{p}<0 .
$$


On the other hand, for all $\varepsilon>0$, there exists $\xi_{3}(p)>0$ such that for all $\xi>\xi_{3}(p)$ and $x \in B_{R-\varepsilon}(0)$

$$
0<\liminf _{t \rightarrow \infty} \frac{1}{t} \log \mathbb{E}\left|u_{t}(x)\right|^{p}<\infty .
$$

In the remainder of this section, we show that the method developed in this paper can be used to study problems with operators other than the fractional Laplacian.

Example 4.3. Consider the following stochastic heat equation with linear drift

$$
\left\{\begin{array}{l}
\partial_{t} u_{t}(x)=-\nu(-\Delta)^{\frac{\alpha}{2}} u_{t}(x)+\lambda u_{t}(x)+\xi \sigma\left(u_{t}(x)\right) \dot{W}(t, x), \quad x \in B_{R}(0), \quad t>0 \\
u_{t}(x)=0, \quad x \in B_{R}(0)^{c}, \quad t>0
\end{array}\right.
$$

where $\lambda$ is a real number and all the other conditions are the same as in (1.3). The mild solution is given by

$$
u_{t}(x)=\left(\mathcal{G}_{D}^{*} u_{0}\right)_{t}(x)+\xi \int_{B_{R}(0)} \int_{0}^{t} p_{D}^{*}(t-s, x, y) \sigma\left(u_{s}(y)\right) F(\mathrm{~d} s, \mathrm{~d} y)
$$

where

$$
p_{D}^{*}(t, x, y)=e^{\lambda t} p_{D}(t, x, y)
$$

and

$$
\left(\mathcal{G}_{D}^{*} u_{0}\right)_{t}(x)=\int_{B_{R}(0)} u_{0}(y) p_{D}^{*}(t, x, y) \mathrm{d} y .
$$

Then there exists $\xi_{0}(\lambda)>0$ such that for all $\xi<\xi_{0}(\lambda)$ and $x \in B_{R}(0)$

$$
-\infty<\limsup _{t \rightarrow \infty} \frac{1}{t} \log \mathbb{E}\left|u_{t}(x)\right|^{2}<0
$$

while for $\varepsilon>0$ there exists $\xi_{1}(\lambda)>0$ such that for all $\xi>\xi_{1}(\lambda)$ and $x \in B_{R-\epsilon}(0)$

$$
0<\liminf _{t \rightarrow \infty} \frac{1}{t} \log \mathbb{E}\left|u_{t}(x)\right|^{2}<\infty .
$$

The proof is very similar to that of Theorem 1.3, we only need to adjust Lemma 2.1 as follows:

$$
\int_{0}^{\infty} e^{\beta t} p_{D}^{*}(t, x, x) \mathrm{d} t \leqslant c_{1}\left[(\lambda+\beta)^{-\frac{1}{\alpha}}+\frac{1}{\mu_{1}-(\lambda+\beta)}\right] .
$$

for all $\beta>0, x \in B_{R}(0)$ provided $0<\lambda+\beta<\mu_{1}$. Now if $x, y \in B_{R-\epsilon}(0)$ and $2\left(\mu_{1}-\lambda\right)+\beta>0$, we have

$$
\int_{0}^{\infty} e^{-\beta t}\left(p_{D}^{*}(t, x, y)\right)^{2} \mathrm{~d} t \geqslant c_{2} \frac{e^{-\left(2 \mu_{1}-2 \lambda+\beta\right) t_{0}}}{2 \mu_{1}+2 \lambda-\beta},
$$

where $c_{1}$ and $c_{2}$ are some positive constants. With some modifications of the proofs above, Theorems 1.3, 1.5, 4.1 and 4.2 hold for the solution of equation (4.1). 
Example 4.4. In this example we consider the generator of a relativistic stable process killed upon exiting $B_{R}(0)$ instead of the fractional Laplacian. We therefore look at

$$
\left\{\begin{array}{l}
\partial_{t} u_{t}(x)=m u_{t}(x)-\left(m^{\frac{2}{\alpha}}-\Delta\right)^{\frac{\alpha}{2}} u_{t}(x)+\xi \sigma\left(u_{t}(x)\right) \dot{F}(t, x), \quad x \in B_{R}(0), \quad t>0 \\
u_{t}(x)=0, \quad x \in B_{R}(0)^{c} .
\end{array}\right.
$$

Here $m$ is some fixed positive number and all the other conditions are the same as in (1.5). We refer the reader to [3] for the needed heat kernel bounds to prove appropriate versions of Theorems 1.3, 1.5, 4.1 and 4.2. We leave it for the reader to fill in the details.

Example 4.5. We conclude this section with this interesting problem. Let $1<\beta<\alpha<2$ and consider the following:

$$
\left\{\begin{array}{l}
\partial u_{t}(x)=-\nu(-\Delta)^{\frac{\alpha}{2}} u_{t}(x)-a^{\beta}(-\Delta)^{\frac{\beta}{2}} u_{t}(x)+\xi \sigma\left(u_{t}(x)\right) \dot{F}(t, x), \quad x \in B_{R}(0), \quad t>0 \\
u_{t}(x)=0, \quad x \in B_{R}(0)^{c} .
\end{array}\right.
$$

Here we refer the reader to [2] for the heat kernel bounds needed to prove suitable versions of Theorems 1.3, 1.5, 4.1 and 4.2 for the solution of the above equation. We leave it for the reader to fill in the details.

\section{References}

[1] Krzysztof Bogdan, Tomasz Grzywny, and Michał Ryznar. Heat kernel estimates for the fractional Laplacian with Dirichlet conditions. Ann. Probab., 38(5):1901-1923, 2010.

[2] Zhen-Qing Chen, Panki Kim, and Renming Song. Dirichlet heat kernel estimates for $\Delta^{\alpha / 2}+\Delta^{\beta / 2}$. Illinois J. Math., 54(4):1357-1392 (2012), 2010.

[3] Zhen-Qing Chen, Panki Kim, and Renming Song. Sharp heat kernel estimates for relativistic stable processes in open sets. Ann. Probab., 40(1):213-244, 2012.

[4] Robert C. Dalang. Extending the martingale measure stochastic integral with applications to spatially homogeneous s.p.d.e.'s. Electron. J. Probab., 4:no. 6, 29 pp. (electronic), 1999.

[5] Mohammud Foondun and Davar Khoshnevisan. Intermittence and nonlinear parabolic stochastic partial differential equations. Electron. J. Probab., 14:no. 21, 548-568, 2009.

[6] Mohammud Foondun and Davar Khoshnevisan. On the stochastic heat equation with spatially-colored random forcing. Trans. Amer. Math. Soc., (365):409-458, 2013.

[7] Mohammud Foondun, Wei Liu, and Erkan Nane. Some non-existence results for a class of stochastic partial differential equations. preprint.

[8] Mohammud Foondun and Eulalia Nualart. On the behaviour of stochastic heat equations on bounded domains. ALEA Lat. Am. J. Probab. Math. Stat., 12(2):551-571, 2015. 
[9] Davar Khoshnevisan. Analysis of stochastic partial differential equations, volume 119 of CBMS Regional Conference Series in Mathematics. Published for the Conference Board of the Mathematical Sciences, Washington, DC; by the American Mathematical Society, Providence, RI, 2014.

[10] John B. Walsh. An Introduction to Stochastic Partial Differential Equations. In École d'été de Probabilités de Saint-Flour, XIV-1984, volume 1180 of Lecture Notes in Math., pages 265-439. Springer, Berlin, 1986.

[11] Bin Xie. Some effects of the noise intensity upon non-linear stochastic heat equations on [0, 1]. Stochastic Process. Appl., 126(4):1184-1205, 2016. 